Bangladesh J. Plant Taxon. 28(1): 277-287, 2021 (June)

(C) 2021 Bangladesh Association of Plant Taxonomists

\title{
CLIMATE CHANGE, BIOSYSTEMATICS AND TAXONOMY
}

\author{
M. KhaIRUL AlaM* \\ Bangladesh Forest Research Institute, Chattogram, Bangladesh \\ Key words: Adaptation, Biodiversity conservation, Phenology, Phenotypic plasticity, \\ Plant functional types, Polyploidy.
}

\begin{abstract}
The history of biosystematics research and its impacts on climate goes before political ramifications. Climate change is altering the environments and likely to result in changes in the distribution of species, flowering times; migrate and adapt to the new environmental conditions; or extinction. Adaptive capacity is the ability of the plants to adapt to the impacts of climate change. Adaptation process is going in nature through phenotypic plasticity, natural selection or migration or polyploidization. The options are not mutually exclusive. Phenotypic plasticity may be the most efficient way of adaptation to a new environment. Polyploidization may increase tolerance to diverse ecological conditions and the high incidence of polyploidy in plants indicates its adaptive significance. Population having polyploid pillar complex is a good backup support towards microevolution and speciation, a mode of adaptation. The paper discusses about these biosystematics approaches towards adaptation to new environmental conditions resulting from climate change. It also discusses about the role of taxonomists under the changed circumstances. It is evident from the review that a set of biosystematics data along with other ecological and conservation information needs to be included in Flora and Monographs. It reveals that it was as far as worked out at the Paris Botanical Congress 1954 and put up by Stebbins in a series of proposals, termed as "Stebbins' Ten Points" that needs further enrichment.
\end{abstract}

\section{Introduction}

Climate change is not a new topic in biology. The study of biological impacts of climate change has a rich history in the scientific literature, since long before there were political ramifications (Parmesan, 2006). In the nineteenth century a tradition was established of study of variation, in nature and in cultivation, of plant species by botanists such as Jordan, Kerner and Bonnier (Davis and Heywood, 1963: 387). Darwin (1859: 78) also attempted to relate the variation to natural selection and stated, "We shall best understand the probable course of natural selection by taking the case of a country undergoing some slight physical change, for instance, of climate. The proportional numbers of its inhabitants will almost immediately undergo a change, and some species will probably become extinct". Long back scientists had the notion of climate migration of plants (Davis and Heywood, 1963: 448) and climatic migration theory for plants presumably dates from Prof. E. Forbes' Theory of Climatic Migrations, published in 1846 (Good, 1931).

Different climatic factors (such as annual and seasonal temperature, annual and seasonal precipitation, atmospheric $\mathrm{CO}_{2}$ concentration) and their interactions act on distribution of different plant species, associations and vegetation types. Clearly, most of these stimuli will be affected directly or indirectly by climate change, and that is likely to result in changes in the distribution of

*E-mail: khairulalam1952@gmail.com 
species, flowering times etc. Rare species will become rarer. The existence of many species in the wild will become threatened because many of them are restricted to a particular range, and because environments will change faster than the most plant species can adapt (Hawkins et al., 2008). Téllez et al. (2007) noted that most species have only a few alternatives in the face of climate change, they can migrate to appropriate environmental conditions; adapt to the new environmental conditions; or become extinct. Evidently, it is expected that the intrinsic capacity of each taxon or group of taxa to respond to climate change will result in different behaviours. Plant diversity provides a buffer against the effects of climate change, and a source of raw materials for adaptation. The impacts of climate change on vegetation, plant population and species have great concern on plant taxonomy and biosystematics.

The name of a plant is the key to information about its use, conservation status, relationships and place within ecosystems (Hawkins et al., 2008) and plant taxonomy deals with naming. Biosystematics is a synthesis process and the taxonomy is the ultimate practice that brings Floras and Monographs. Taxonomy and systematics is one of the oldest branches of biology and the Linnaean system of classification that is still used today dates back to the 1750s. Throughout its history taxonomy has continuously reinvented itself, but today the science is experiencing an unprecedented rate of change. The emerging interest for adaptation of plants for climate change is representing a vital input to taxonomy. This paper attempts to assess the changes in plants' adaption for climate change and areas that plant taxonomy faces in resolving the emerging issues.

\section{Climate change and plant adaption}

In recent decades a great deal of research is being undertaken to assess the effects of climate change on the distribution and abundance of individual species (Carey, 1996; Parmesan, 1996). This is based on the theory that ultimately the distribution of a species is limited by its physiological responses to climate. One of the earliest exponents of this theory was Ronald Good's "Theory of Tolerance" (Good, 1931). Evidence from palynology and fossil records have shown that, in the past, plant species have successfully responded to environmental change by 1) remaining within the modified climate by tolerance or adaptation, 2) migrating to track suitable conditions, or most likely a combination of both (Engler et al., 2009). Climate change is altering the environments in which all organisms develop. Plant species can adjust to these novel conditions through phenotypic plasticity, adapt through natural selection or migrate to follow conditions to which they are adapted; these options are not mutually exclusive.

\section{Phenotypic plasticity}

For any given plant species or population, determining responses to environmental changes will require an understanding of the environmentally induced variation in the phenotype of individual plants (Nicotra et al., 2010). Phenotype is simply the manifestations of genotype + environment (Stace, 1980). Works on plasticity forms some of the basis of earliest biosystematics experiments and dates back to nineteenth century. G. Bonnier carried out a wide range of cultivations experiments in 1884 in the Alps and the Pyrenees (Stace, 1980). Turesson was the pioneer in the field of biosystematics. Since 1916 Turesson, initiated experimentation and formulated the ecotype concept (Turesson, 1922 as cited in Davis and Heywood, 1963). His studies revealed that plant populations, even of the broadly ranging species, are often adapted to local environmental condition.

Plasticity usually refers to environmentally influenced variability in a particular life-stage, or (in plants and colonial invertebrates) to variation in the behaviour, form, physiology, or sequence of modules produced at a particular stage of growth (West-Eberhard, 1989). It can play a major role in both the ecological distribution of organisms and their patterns of evolutionary 
diversification. Taxa consisting of adaptively plastic genotypes may inhabit a broad range of environmental conditions (Sultan, 2003; Chambell et al., 2005).

Phenotypic plasticity may be the most efficient way in which a plant adapts itself rapidly to a new environment. Many plants are able to adapt and grow in a wide range of environments. Much of the phenotypic variations in many organisms are the result of the plastic responses of the individuals to factors of the environment. Such variations are termed as phenotypic modifications, and this phenomenon is known as phenotypic plasticity, varies greatly in different species (Heywood, 1976). Stace (1980) emphasized the importance to recognize plasticity by taxonomists. Davis and Heywood (1963) termed these phenotypic environmental modifications as phenecotypes, and suggested to include their range of variations in the description of the taxon. Identification of phenecotypes by taxonomists for particular changed environment will help in conservation.

Both adaptive and non-adaptive plasticity will play a role in the context of plant responses to climate change (Nicotra et al., 2010). Species with a greater adaptive plasticity may be more likely to survive in novel environmental conditions, since such changes typically occur too rapidly to allow for an evolutionary (or in some cases a migratory) response (Gratani, 2014). "Plasticity" and "development" are related terms that are becoming increasingly common in evolutionary biology and ecology. Both phenomena have passed through a period of neglect (West-Eberhard, 1989). Bradshaw (1965, as cited in West-Eberhard, 1989) noted that botanists were carefully avoiding any mention of plasticity; environmental effects in experiments were considered "only an embarrassment." In an environment rapidly changing on local and global scales, narrowly adapted populations with low plasticity in selectively important characters might be at a higher risk of extinction. It is evident from various studies that climate change favour high level of phenotypic plasticity in plants (Parmesan, 2006; Valladares et al., 2007) what the plant taxonomy today should take into consideration.

\section{Polytopy and distribution range}

One of the climate change impacts is likely to be the changes in species' ranges. We already have in-depth understanding of how some species in some parts of the world have moved in the past in response to changing climates and this is being employed to consider what future changes might occur (Stace, 1980). When a species occurs in two or more separate areas, it is said to be polytopic (Davis and Heywood, 1963). The most frequent explanations of polytopy are that disjunct areas have been bridged in the past either by long-distance dispersal or by slow migration. The latter explanation of slow migration being known as climatic migration theory presumably dates from Prof. E. Forbes' Theory of Climatic Migrations, published in 1846 (Good, 1931). According to Good's theory of tolerance, a species is able to exist and reproduce successfully within a definite range of climatic and edaphic conditions. Further, an understanding of the climatic tolerances of species seems attainable by matching current distributions with key climatic variables. The resulting bioclimatic envelope gives some idea of species' climatic tolerances (Harris et al., 2006). Polytopic differentiation of species or units at lower rate almost certainly occurs (Davis and Heywood, 1963), and seems to be a good mode of adaptation of plants to climatic changes.

Future changes in climate could result in extinctions, range shifts, changes in major vegetation types and alterations in feedbacks between vegetation and the atmosphere. Indeed, the distribution of many plant species has already altered in response to climate change; some species have shown up to $6 \mathrm{~km}$ pole ward migration each year over the past 16-132 years (Parmesan and Yohe, 2003, as cited in Nicotra et al., 2010). 
Observations of range shifts in parallel with climate change have been particularly rich in northern European countries, where observational records for many birds, butterflies, herbs, and trees date back to the mid-1700s. Since the early part of the twentieth century, researchers have documented the sensitivity of insects to spring and summer temperatures (Parmesan, 2006).

The change in climate in the coming years will lead to the extinction of some species and appearance of new ones. Broad-niched species will dominate, while narrow-niched ones would be eliminated. Thus, we have to go further in investigating the change in our flora and how much our plants can cooperate with environmental disorders (Taia, 2005), a great concern of plant taxonomy.

Climate change will place pressure on the natural range and survival of wild populations of plants. We are to understand plant diversity and the way of both internal and external adaptation according to environmental changes, and that needs attention of plant taxonomy.

\section{Phenology}

Ecological changes in the phenology and distribution of plants and animals are occurring due to change in climatic conditions. Phenology is the study of recurring seasonal events, such as flowering and leaf falling in plants and hibernation and migration in animals (Hawkins et al., 2008). Parameters such as the date and duration of flowering can be compared with climatic parameters such as temperature, rainfall and humidity to see if there is any correlation (http://www.rbge.org.uk/science/plants-and-climate-change/phenology-projects). Linnaeus in his Philosophia Botanica listed dates for first flowering, leafing, fruiting and leaf falling, and recommended that 'Floral calendars should be completed every year in every province, according to the leafing, flowering, fruiting and leaf-shedding, with simultaneous observations of the climate, so that it may be ascertained how regions differ among themselves' (Linnaeus, 1751, as cited in Haper et al., 2004). Phenological research at Royal Botanic Garden Edinburgh dates back to the 1850s, when the Curator, James McNab first recorded the flowering dates of more than 60 species (Harper et al., 2004). Evolutionary adaptations to warmer conditions have occurred in species' ranges, and resource use and dispersal have evolved rapidly at expanding range margins (Parmesan, 2006).

Phenological studies will increase the understanding of the mechanism in plants which responds to climate changes and will enable scientists to predict how plants will respond to these climatic changes. The locality information that accompanies each herbarium specimen would be a valuable source of information regarding the placement of permanent plots (Gallagher et al., 2009). However, errors may arise from using of herbarium materials through unresolved taxonomic issues, misidentification or nomenclatural inconsistencies between organisations. These should be taken into consideration and tried to be minimized.

Despite of limitations, phenological studies have important consequences for conservation, agriculture, horticulture and forestry; what gardeners are able to grow in the future will, without doubt, be influenced by climate change. The range of flowering and fruiting time is recorded in Flora and Monographs. We should be more specific to monitor impacts of climate change on plant communities with these attributes.

\section{Polyploidization}

Polyploidization results in multiplication of the genome and an increase in gene content that frequently leads to morphological and physiological differences between polyploids and their diploid progenitors. Polyploidy, or whole genome duplication (WGD), is now recognized as a major evolutionary force not only in plants, but also in all eukaryotes (Soltis et al., 2014). WGD generally results in instant speciation, increasing biodiversity and providing new genetic material 
for evolution (Levin, 1983). The high incidence of polyploidy in plants indicates that it may have important adaptive significance (Tate et al., 2005). There are a number of factors that may provide polyploids with adaptive and evolutionary advantages. Perhaps most importantly, polyploids can be significantly more heterozygous than their diploid counterparts. The degree of heterozygosity may be a key factor in the growth, performance, and adaptability of a polyploid.

The influence of polyploidy on adaptability and resistance to biotic and abiotic stresses has been widely studied in crop plants (Levin, 1983). Polyploids are more frequent at higher elevations, higher latitudes and may be more tolerant to dry conditions, suggesting a fitness advantage for polyploids under those environments. A study sampling 640 endangered and 81 invasive species worldwide has led to the conclusion that endangered species tend to be diploids while invasive species tend to be polyploids, suggesting that polyploidization may increase tolerance to diverse ecological conditions (Pandit et al., 2011).

Polyploidy can also be an important factor in species invasion success through a combination of 'pre-adaptation'; and the possibility for subsequent adaptation due to a larger genetic diversity that may assist the 'evolution of invasiveness' (te Beest et al., 2012). Polyploidy may drive a population into a new 'adaptive sphere' and allow occupying habitats that are beyond of its progenitors (Levin, 1983; Bayer, 1998). It, particularly when accompanied by hybridization between either different species or different ecotypes of same taxon, is one of the quickest ways for new species or races to become adapted to different ecological circumstances (Stebbins, 1984). Having multiple ploidy levels, each with its own habitat preference, may thus favour a species in colonizing larger geographic ranges with heterogeneous environmental conditions (Ramsey, 2011; te Beest et al., 2012; Hao et al., 2013). Apart from the discussion about whether polyploids have wider distributions, given the influence of polyploidy on plant growth, physiology, gene expression, etc., it is likely that niche differentiation readily occurs between different ploidy levels. Cyto-geographical studies show that diploids and polyploids often occupy different parts of the landscape and that polyploids typically replace their diploid parents along ecological gradients, such as moisture gradients, with polyploids generally occupying drier habitats than diploids (te Beest et al., 2012). Polyploidy may propel a population into a new 'adaptive sphere' given the myriad changes that accompany genome doubling (Soltis et al., 2014.).

Quite often morphologically similar units consist of a series of distinct diploid species which has hybridized and become polyploids to produce a range of teraploids, hexaploids and sometimes higher level of ploidy. Because of the combination of genomes the distinction between taxa at diploid level blurred with tetraploid and higher levels cannot be recognized. Such a situation is described as polyploid pillar complex (Davis and Heywood, 1963; Stace, 1980). Population having such complex is a good backup support towards microevolution and speciation, a mode of adaptation.

Polymorphism may be of evolutionary significance and sometimes worthy of taxonomic recognition (Valentine, 1975) but taxonomists have paid little attention to it (Stace, 1980). Every taxonomist in the course of studies wants to know the variation, but because of continuity tries to avoid the variations in documentation process.

\section{Plant functional types (PFTs)}

It is being increasingly realized that, in order to understand the interaction of plants and ecosystem processes and their potential response to global environmental changes, groups of species with shared characteristics, known as plant functional types (PFTs), need to be identified (Duckworth et al., 2000). The concept of plant functional types has a long history, and Von Humboldt in 1806 first offered the first widely recognized classification of physiognomic plant types following his travels in South America (Duckworth et al., 2000). The most important early 
system of functional classification is life-forms system of Raunkier (Duckworth et al., 2000) and the adaptation of plants to environmental conditions is to some extent expressed in life-form classifications (Davis and Heywood, 1963). These groupings of plant species on the basis of common biological parameters reduce a wide diversity of species to small number of functional groups, which enables the identification of general principles for the functioning of organisms and can be used for making predictions (Duru et al., 2009).

PFTs provide a valuable tool for the understanding of ecological and biogeographical processes and the prediction of change. This is particularly important in regions where the flora remains largely unknown. Functional classifications often cut across taxonomic classifications and may be more meaningful in terms of plant response, in addition to overcoming certain problems associated with the traditional taxonomic species-based approach. They are thus likely being more useful than taxonomic groupings in the interpretation of plant response and resource use. Plant functional types are derived from traits based on species morphology, physiology and/or life history, depending on the aims and scale of the research. To enhance the end use values of taxonomic products like Local Floras, PFTs need to be assessed and incorporated.

\section{Plant functional types and climate change}

Research into the impacts of future climate change is usually considered best carried out at the individual species level. Since it will not be possible to develop models and make predictions for every species, Woodward and Cramer (1996) used the PFT approach to assess sensitivity and predict responses to several aspects of climate change (Díaz and Cabido, 1997). There is a recognized relationship between PFTs and climate, particularly at the global or biome scale and PFTs seem a promising tool for predicting the direction and rate of changes in vegetation in the face of climate change (Duckworth et al., 2000). Responses of PFTs to climate change are determined by specific trait characteristics (Esther et al., 2010) and concerns a deal with plant taxonomy.

\section{Conservation strategy}

Global climate change may have a serious impact on genetic resources in tropical forest trees. Genetic diversity plays a critical role in the survival of populations in rapidly changing environments. Furthermore, most tropical plant species are known to have unique ecological niches, and therefore changes in climate may directly affect the distribution of biomes, ecosystems, and constituent species. Climate change may also indirectly affect plant genetic resources through effects on phenology, breeding systems, and plant-pollinator and plant seed disperse interactions, and may reduce genetic diversity and reproductive output. As a consequence, population densities may be reduced leading to reduction in genetic diversity through genetic drift and inbreeding (Bawa and Dayanandan, 1998). So, identification and conservation of populations with polyploid complex is a concern of biosystematics.

Tropical forest plants may respond to climate change through phenotypic plasticity, adaptive evolution, migration to suitable site, or extinction. However, the potential to respond is limited by a rapid pace of change and the non-availability of alternate habitats due to past and present trends of deforestation. Thus climate change may result in extinction of many populations and species. Our ability to estimate the precise response of tropical forest ecosystems to climate change is limited by lack of long-term data on parameters that might be affected by climate change. Collection of correlative data from long-term monitoring of climate as well as population and community responses at selected sites offer the most cost-effective way to understand the effects of climate change on tropical tree populations. However, mitigation strategies need to be 
implemented immediately. Taxa that are in verge of extinction should be identified and protected through both in-situ and ex-situ conservation programs.

In the near future it will be important to collect data by working in the field and, in particular, in primary forests and/or in well-conserved habitats where new stress factors are limited in order to define standard protocols useful for comparative studies in integrated approach in plasticity. Among the strategies, environment conservation should protect heterogeneity between and within habitats in order to maintain larger intra-specific variability and, thereby conserving a variety of phenotypic specializations that will be able to buffer future environmental extremities due to climate and land-use changes (Wellstein et al., 2013).

Since species with extensive geographical range have the potential to exhibit large intraspecific variations in physiology, morphology, and phenology, they may be good models for the study of local and regional adaptations (Gratani, 2014). Further research on this issue regarding forest tree species is needed in order to understand the impact that global climate change may have on the existing populations. Phenotypic plasticity is of prime importance to define the ability of the target genotypes to respond to new conditions, and therefore to decide on the best conservation strategies to be applied (Chambel et al., 2005).

\section{Role of plant taxonomy}

As we are in the twenty-first century, the demand for taxonomy is greater than ever before. The global imperative for the conservation of biodiversity has brought into sharp focus both the need for and the needs of taxonomic research. It will also demand a disciplined and concerted effort to balance the investment of scientific resources among different themes within plant diversity research. But to contribute effectively to these, plant taxonomists must broaden the way they currently see their discipline. With changed perspective and mindset, plant taxonomists can be able to actively contribute to these global and national agendas.

The change in environments in the coming years will lead to the extinction of some species and appearance of new ones. Broad-niched species will dominate, while narrow-niched ones will be eliminated. Thus, we have to go further in investigating the change in our flora and how much our plants can cooperate with environmental disorders (Taia, 2005).

D'Andrea et al. (2009) pooling herbarium data of 24 herbaria from 15 European countries and literature data on floristic investigation from 25 European countries found that the distribution range of Lactuca serriola (Asteraceae), a species native to the summer-dry Mediterranean climate, has expanded northwards during the last 250 years. Their work highlights the importance of historical herbarium data for expansion of habitat due to the influence of climate change. Herbaria the repository of such historical distributional data can provide valuable information for planning management of contemporary environmental problems such as species responses to environmental change.

Species of contrasting functional traits and plasticities co-occur in many ecosystems. However, our understanding of the functional implications of phenotypic plasticity in multifactor environments (and all environments are multifactor to one extent or another) is limited. The real adaptive value of plasticity of woody plants in a global change scenario is contingent on the given combination of factors that operates in each habitat, and clearly deserves more attention due to its intrinsic complexity (Chambel et al., 2005).

In a rapidly changing environment on local and global scales, narrowly adapted populations with low plasticity in selectively important characters might be at a higher risk of extinction. In the near future it will be important to collect data by working in the field and, in particular, in primary forests and/or in well-conserved habitats where new stress factors are limited in order to define 
standard protocols useful for comparative studies. Among the strategies, environment conservation should protect heterogeneity between and within habitats in order to maintain larger intraspecific variability and, thereby conserving a variety of phenotypic specializations that will be able to buffer future environmental extremities due to climate and land-use changes (Wellstein et al. , 2013).

The outputs of research in taxonomy and biosystematics have always had a broad range of end-users. Taxonomy was a much applied service science, but as decades went by, less and less attention seems to have been paid to the precise needs of changing consumers (Heywood, 1983). Over the last three decades there have been huge changes in the way environmental scientists seek to understand and protect the natural world. The Convention on Biological Diversity was signed at the Earth Summit in 1992 and was reframed in 2010. National and international targets to protect biodiversity have been instituted, many including statutory obligations. Over the same time period and in particular spurred by the Millennium Ecosystem Assessment, new thinking based around the concept of ecosystem services has come to the fore. The resource-based economic regime is now in place, in which taxonomy is an integral component in terms of biodiversity protection, remediation, eco-development, product development and quality evaluation (Nair, 2004). Thus, major constituencies now have been environmental scientists, novel product development enterprises and eco-development agencies.

\section{Local Floras need more information flow}

Analysing 30 local Floras from 1738 to 2002, Hill (2003) stated that in spite of Local Floras contain accounts of the environment; information on the distribution and abundance of plants, but there is little flow of information between ecologists and flora writers. Hill's possible explanations are the disparity in the spatial scale of interest, the subtlety of plant life histories, the obscurity of key environmental factors, and the emphasis of floras on rarities rather than on the ecosystem processes. Local floras are used by ecologists to a small extent, mainly to provide information on distributional change, and ecological information is included as background in some local floras but not in others (Hill, 2003).

One of the concluding remarks by Stace in 1980 for the end of past century that "The further broadening of the application of very wide range of taxonomic characters to all groups of plants." still remains valid at the end of the second decade of the current century.

Heywood (1984) considering the design of Floras for the future two interrelated aspects have to be considered: (1) the purpose of Flora and audience aimed at, (2) technical aspects of data presentation in a computer oriented world.

A critical analysis of data- needs of environmentalists, conservation biologists and land use planners in changed circumstances are mostly ecological, gen-ecological and eco-physiological. A glance at the accounts written for the Biological Flora of the British Isles shows, that they make extensive use of descriptions of vegetation, but that local floras are little cited. The Ecological Database of the British Isles has been constructed from a wide variety of sources and consists of a suite of over 130 ecological and morphological characteristics, and vice-county distribution in Britain, European distribution by country. The data are obtained from the literature and therefore coverage varies greatly between species (Fitter and Peat, 1994). If we make an overview of biosystematic information it reveals that most of the needs of today's users of taxonomy concerned with world changes are there, what Stebbins rightly made in this series of proposals in about seven decades back.

A list of kinds of biosystematic data which monographers should become informed about as far as possible was worked out at the Paris Botanical Congress 1954, and put up by Stebbins in a 
series of meetings in the form of a series of proposals. These proposals termed as Stebbins' Ten Points (Davis and Heywood, 1963) are:

1. Data on variability in terms of population

2. Chromosome numbers adequately obtained and recorded

3. Reproductive biology

4. Apomixis

5. Structural heteozygosity

6. Presence and type of polyploidy

7. Degree to which species can be crossed

8. Presence and extent of introgression

9. Detection of homoploid population or species of hybrid origin

10. Habitat

It was further suggested that Flora and Monographs indications where possible by word or symbols. The practice of taxonomy involves making decisions on materials and resources available. So, preservation of good voucher herbarium specimens is always needed which allow comparisons to reach to a good decision. Notes on descriptions and analysis of environment conditions as far as possible will help identifying the changing scenarios. Thus it is evident that there is no alternative of elaborate field notes and field work what is always an accompaniment of field taxonomists.

Taxonomy has a multitude of end-users, from scientists through applied biologists and to amateur naturalists, and ultimately to the general public. Few other sciences have such a broad potential audience. So, whatever product we are producing we are to make them field and practical oriented.

Thus, major constituencies now have been environmental scientists, novel product development enterprises and eco-development agencies. It will also demand a disciplined and concerted effort to balance the investment of scientific resources among different themes within plant diversity research. But to contribute effectively to these, plant taxonomists must broaden the way they currently see their discipline. With changed perspective and mindset, plant taxonomists can be able to actively contribute to these global and national agendas.

\section{References}

Bawa, K.S. and Dayanandan, S.D. 1998. Global Climate Change and Tropical Forest Genetic Resources. Climatic Change 39(2): 473-485. DOI: 10.1023/A:1005360223639

Bayer, R.J. 1998. New perspective into the evolution of polyploidy complexes. In: van Raamsdonk, L.W. D. and den Nijis, J.C.M. (Eds.). Plant Evolution in Man-made Habitats: Proceedings of $7^{\text {th }}$ IOPB, Amsterdam, 1998, pp. 359-373.

Bradshaw, A. D. 1965. Evolutionary significance of phenotypic plasticity in plants. Adv. Genet. 13: 115-55.

Carey, P.D. 1996. DISPERSE: a cellular automaton for predicting the distribution of species in a changed climate. Global Ecology and Biogeography Letter 5: 217-226.

Chambel, M.R., Climent, J., Alía, R. and Valladares, F. 2005. Phenotypic plasticity: A useful framework for understanding adaptation in forest species. Invest Agrar: Sist. Recur. For. 14(3): 334-344.

D’Andrea , L., Broennimann , O., Kozlowski , G., Guisan, A. , Morin, X., Keller-Senften , J. and Felber, F.N. 2009. Climate change, anthropogenic disturbance and the Northward range expansion of Lactuca serriola (Asteraceae). J. Biogeography. 36(8):1573-1587.

Darwin, C. 1859. The Origin of Species by means of Natural Selection. London.440 pp.

Davis, P.H. and Heywood, V.H. 1963 (Reprinted 1973). Principles of Angiosperm Taxonomy. Robert E. Krieger Publishing Company, New York. 558 pp. 
Diaz, S. and Cabido, M. 1997. Plant functional types and ecosystem function in relation to global change. Journal of Vegetation Science 8: 463-474.

Duckworth, J.C., Kent, M. and Ramsay, P.M. 2000. Plant functional types: an alternative to taxonomic plant community description in biogeography? Progress in Physical Geography 24(4): 515-542.

Duru, M., Khaled, R.A.H., Ducourtieux. C., Theau, J.P., de Quadros, F.L.F. and Cruz, P. 2009. Do plant functional types based on leaf dry matter content allow characterizing native grass species and grasslands for herbage growth pattern? Plant Ecol. 201: 421-433.

Engler, R., Randin, C. F., Vittoz, P., Cza'ka, T., Beniston, M., Zimmermann, N. E. and Guisan, A. 2009. Predicting future distributions of mountain plants under climate change: does dispersal capacity matter? Ecography 32: 34-45. DOI: 10.1111/j.1600-0587.2009.05789.

Esther, A., Groeneveld, J, Enright, N.J., Miller, B.P., Lamont, B.B., Perry, G.L.W., Blank, F.B. and Jeltsch, F. 2010. Sensitivity of plant functional types to climate change: Classification tree analysis of a simulation model. Journal of Vegetation Science 21: 447-461.

Fitter, A.H. and Peat, H.J. 1994. The Ecological Flora Database, J. Ecol. 82:415-425. http://www. ecoflora.co.uk

Gallagher, R.V., Hughes, L. and Leishman. M.R. 2009. Phenological trends among Australian alpine species: using herbarium records to identify climate-change indicators. Australian Journal of Botany 57: 1-9.

Good, R.D.O. 1931. A theory of plant geography. The New Phytologist 30 (3): 149-171.

Gratani, L. 2014. Plant Phenotypic Plasticity in Response to Environmental Factors. Volume 2014, Article ID 208747, 17 pages. http://dx.doi.org/10.1155/2014/208747.

Hao G.Y., Lucero, M. E., Sanderson, S. C., Zacharias, E.H. and Holbrook, N. M. 2013. Polyploidy enhances the occupation of heterogeneous environments through hydraulic related trade-offs in Atriplex canescens (Chenopodiaceae). New Phytologist 197: 970-978.

Harper, G., Mann, D. and Thomson, R. 2004. Phenological Monitoring at Royal Botanic Garden Edinburgh. Sibbaldia: The International Journal of Botanic Garden Horticulture 2: 33-45.

Harris, J.A., Hobbs, R.J., Higgs, E. and Aronson, J. 2006. Ecological restoration and global climate change. Restoration Ecology 14 (2): 170-176.

Hawkins, B., Sharrock, S. and Havens, K. 2008. Plants and climate change: which future? Botanic Gardens Conservation International, Richmond, UK.

Heywood, V.H. 1976. Plant Taxonomy (second edition). Edward Arnold, London.

Heywood, V.H. 1983. The Mythology of Taxonomy. Trans. Bot. Soc. Edin. 44: 79-84.

Heywood, V.H. 1984. Floras for the future. In: Heywood, V.H. and Moore, D. M. (Eds.) Current Concepts in Plant Taxonomy, Sytematics Association Special Volume No. 25, ed.. Academic Press, London, pp. 397-410.

Hill, M.O. 2003. Using data from local floras for ecological research. Watsonia 24: 321-329

Levin, D.A. 1983. Polyploidy and novelty in flowering plants. Amer. Naturalist 122: 1-25.

Linnaeus, C. 1751. Philosophica Botanica. Oxford University Press Edition, 2003, 402 pp.

Nair, P.K.K. 2004. Plant Taxonomy. Current Science. 86(5): 665-667.

Nicotra, A.B., Atkin, O.K., Bonser, S.P., Davidson, A.M. , Finnegan, E.J., U. Mathesius, Poot, P. Purugganan, M.D. Richards, C.L., Valladares, F., and van Kleunen. M. 2010. Plant phenotypic plasticity in a changing climate. Trends in Plant Science 15(12):684-692.

Pandit M.K., Pocock, M.J.O., Kunin W.E. 2011. Ploidy influences rarity and invasiveness in plants. J. Ecol. 99: $1108-1115$.

Parmesan, C. 1996. Climate and species' range. Scientific correspondence. Nature 382: 765-766 [https:// www.nature.com/articles/382765a0]

Parmesan, C. 2006. Ecological and Evolutionary Responses to Recent Climate Change. Annu. Rev. Ecol. Evol. Syst. 37: 637-669.

Parmesan, C. and Yohe, G. 2003. A globally coherent finger print of climate change impacts across natural systems. Nature 421: 37-42. 
Ramsey, J. 2011. Polyploidy and ecological adaptation in wild yarrow. Proc. Natl. Acad. Sci. USA. 108(17): 7096-7101. doi: 10.1073/pnas. 1016631108

Soltis, D.E., Clayton. J.V. and Soltis, P.S. 2014. The polyploidy revolution then...and now: Stebbins revisited. Am. J. Bot. 101 (7): 1057-1078.

Stace, C. 1980. Plant Taxonomy and Biosystematics. Edward Arnold. 279 pp.

Stebbins, G.L. 1984. Polyploidy and the distribution of the arctic-alpine flora: new evidence and new approach. Bot. Hel. 94: 1-13.

Sultan, S. E. 2003. Phenotypic plasticity in plants: a case study in ecological development. Evolution \& Development 5 (1): 25-33

Taia, W.K., 2005. Modern Trends in Plant Taxonomy. Asian Journal of Plant Sciences 4:184-206.

Tate, J.A., Soltis P.S., Soltis D.E. 2005. Chapter 7 - Polyploidy in Plants. In: T. Ryan Gregory. (Ed.) The Evolution of the Genome. Academic Press, pp. 371-426.

te Beest, M., Le Roux, J. J., Richardson, D.M., Brysting, A.K., Suda, J., Kubešova, M. and Pyšek, P. 2012. The more the better? The role of polyploidy in plant invasions. Annals of Botany 109(1): 19-45 doi:10.1093/aob/mcr277.

Téllez, O., Dávila, P., Ayala, M., Gutiérrez, K. and Melchor, I., 2007. Case studies on the effect of climate change on the flora of Mexico. BGjournal 4(2): 17-21.

Turesson, G. 1922. The genetotypic response of the plant species to the habitat. Hereditus 3:211 -350.

Valentine, D.H. 1975. The taxonomic treatment of polymorphic variations. Watsonia 10: 385390.

Valladares, F., Gianoli, E., and Gómez, J.M. 2007. Ecological limits to plant phenotypic plasticity. New Phytologist 176: 749-763.

Wellstein, W., Stefano, C., Giaandiego, C., Sander, B., Marco, G., Francesco, S. and Roberto, C. 2013. Intraspecific phenotypic variability of plant functional traits in contrasting mountain grassland habitats. Biodiversity and Conservation 22(10): 2353-2374.

West-Eberhard, M.J. 1989. Phenotypic plasticity and the origins of diversity. Annu. Rev. Ecol. Syst. 20:24978.

Woodward, F.I. and Cramer, W. 1996: Plant functional types and climatic changes: introduction. Journal of Vegetation Science 7: 306-308. 